\title{
A CASE STUDY ON AYURVEDIC MANAGEMENT OF DADRU W.S.R TO TINEA CRURIS
}

\author{
Dr. Dnyaneshwar Kantaram Jadhav 1 ه
}

${ }^{1}$ M.D Kaychikitsa (Ayu). Assistance Professor, Kaychikitsa Department, Shri Dhanwantri Ayurvedic Medical College and Research Centre, India
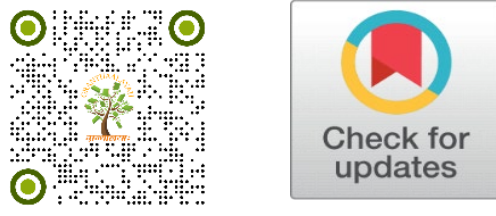

Received 15 April 2021

Accepted 1 May 2021

Published 1 July 2021

\section{CorrespondingAuthor}

Dr. Dnyaneshwar Kantaram Jadhav, dj85015@gmail.com

DOI 10.29121/jahim.v1.i1.2021.3

Funding: This research received no specific grant from any funding agency in the public, commercial, or not-for-profit sectors.

Copyright: (C) 2022 The Author(s). This work is licensed under a Creative Commons Attribution $\quad 4.0$ International License.

With the license CC-BY, authors retain the copyright, allowing anyone to download, reuse, re-print, modify, distribute, and/or copy their contribution. The work must be properly attributed to its author.

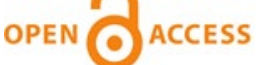

\section{ABSTRACT}

Ayurved is time tested science. From ancient to present era its principles never change. With great logics its cure all types of disease.

Tinea cruris is fungal infection of the groin region.it is ring like shape with any size. It is found more in male then female. According to ayurved all skin disease comes under kushta vhydhi. Tinea cruris can be correlated with dadru. Dadru is one of the Kshudrakustha with predominance of Pitta and Kapha dosha.

31 years old male suffering from Elevated ring like patch around buttock region, Severe itching, Discoloration/Redness, Burning sensation since last 8 months. He took Modern medicine but no relief from that. With Modern medicine he just got relief from itching, that also reoccur again during nighttime.

Ayurvedic medicines along with good hygiene and some lifestyle modifications give best result.

Keywords: Tinea Cruris, Dadru, Ayurved

\section{INTRODUCTION}

Tinea cruris is fungal infection of the groin region. Eczema marginatum, crotch itch, dhobi itch, gym itch, jock itch are other names of this disease. It is also called as dermatophytosis. Tinea cruris is found in all age and sex. It is observed that males are infected more than females. Ring like rash is clearly identification of this disease. Its patch is typically red or brown ring like structure, with irritation and. Its size can be small, medium, or large too. Andhare (2019)

In Ayurved it can correlated with dadru. There is no separate description of Nidana Panchak of Dadru. The Samanya Nidana, Poorvarupa mentioned for Kusta can be apply for dadru. 


\section{Table 1}

Table 1 Showing Comparative Symptoms of Tinia Cruris and Dadru. Andhare (2019)

\begin{tabular}{ccc} 
S. No & Symptoms of Tinea Curis & Symptoms of Dadru Kushta \\
\hline 1 & Erythema & Raga \\
2 & Itching & Kandu \\
3 & Granular surface & Pidka \\
4 & Circular elevation of skin & Utsanna mandal \\
\hline
\end{tabular}

\section{A case report as follow}

A 31-year-old male patient came to us with chief compliant of (3)-

1) Elevated ring like patch around buttock region.

2) Severe itching +++

3) Discoloration/Redness ++

4) Burning sensation +++

Patient had above complaints for 8 months.

No H/o / HTN, Asthma

\subsection{HISTORY OF PERSONAL ILLNESS}

31 years old male suffering from Elevated ring like patch around buttock region, Severe itching, Discoloration/Redness, Burning sensation since last 8 months. He took Modern medicine (Tab.Allegra $120 \mathrm{mg}$, Tab.levocet 4mg, Tab. fluconazole 150 mg, fourderm cream) but no relief from that. With all this Modern medicine he just got relief from itching, that also reoccur again during night-time. Discoloration/Redness increased gradually, then he decides to take Ayurved treatment. For Ayurvedic treatment he came to our clinic - Nakshtra Ayurved Panchkarma Clinic \& Research Center, Mumbai.

\subsection{PERSONAL HISTORY}

Occupation: Engineer.

\subsection{ASHTAVIDHA PARIKSHANA}

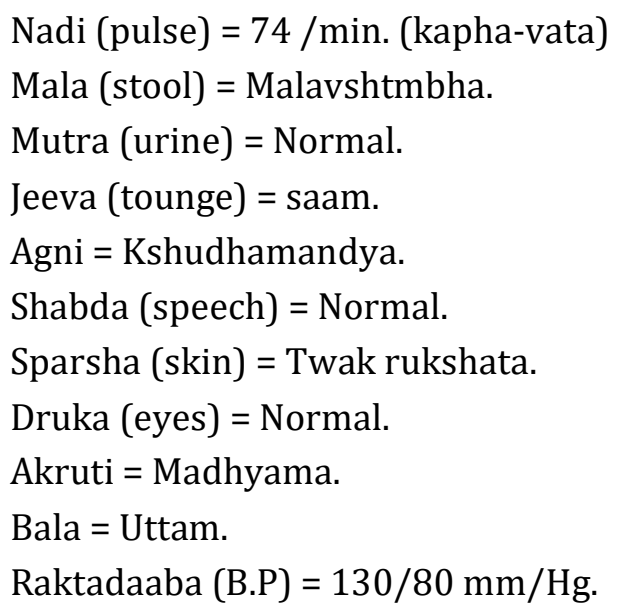




\section{MATERIAL AND METHOD}

\subsection{MATERIAL}

\section{Table 2}

\begin{tabular}{|ccccc}
\hline \multicolumn{2}{l}{ Table 2 Showing Material Used for Study } \\
S. No & Dravya & Dose & Duration & Anupana \\
1 & Gandhak Rasayan vati & $250 \mathrm{mg}$ & Twice a day & Lukewarm water \\
2 & Arogyavardhini vati & $500 \mathrm{mg}$ & Twice a day & Lukewarm water \\
3 & Pachak vati & $250 \mathrm{mg}$ & Twice a day & Lukewarm water \\
4 & Gandahrva haritaki vati & $500 \mathrm{mg}$ & H. S & Lukewarm water \\
5 & Mahamanjishtadhi Kashaya & $20 \mathrm{ml}$ & Twice a day & Lukewarm water \\
\hline 6 & Panchawalkal oilment & For local application: Twice a day \\
\hline 7 & Avagahana (Triphala + Khadir + Nimba) & \multicolumn{3}{c}{ At morning } \\
\hline
\end{tabular}

\subsection{METHOD}

Centre of study: Nakshtra Ayurved panch karma clinic \& research center, Mumbai.

Type of study: Simple random single case study.

\section{DISCUSSION AND RESULT}

\subsection{RESULT}

Clinical examination of the patients revealed regression of all symptoms within 7 days.

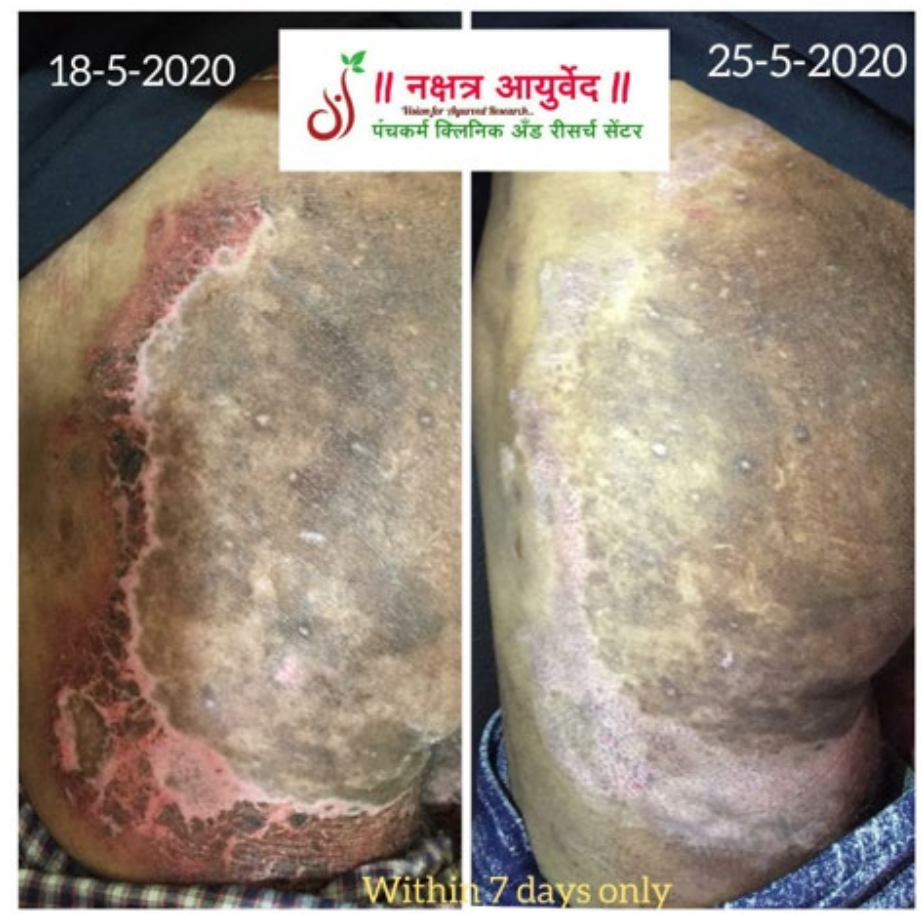




\subsection{DISCUSSION}

\section{Hetu of Dadru (Tinea cruris)}

1) Ahar

- Improper and irregular diet.

- Chiken, Matsya sevan (daily)

- Dadhi (on each alternate day)

- $\quad$ Biscuit (daily with tea - daily)

- All types of fast-food

2) Vihar

- Prolong seating

- Ratri jagran.

- Bath (twice a week)

3) Manasika nidan- vegavrodha causes vata vrudhi.

\section{SANPRAPTI GHATAK}

Dosh: tridoshaj (kapha-pitta pradhana)

Dushya: Twak, Rakta, Lasika (Ambu), Sweda

Srotus: Rasa, Rakta.

Adhishthana: twacha

Rogamarga: bahya

\section{SAMPRAPTI PURIPANDA AND RAJU (2016)}

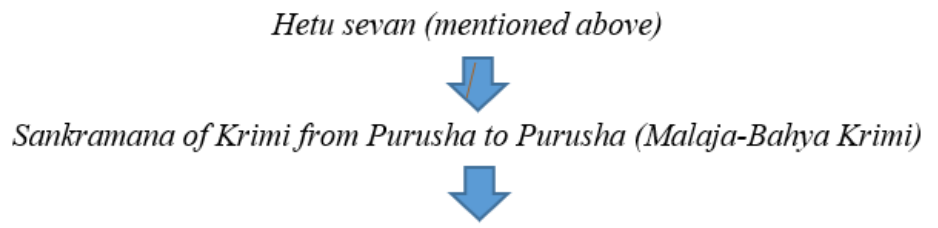

That Krimi entered into Twak through Sweda and vitiates Tridosha, Twak, Raktha and Lasika

Kandu, Pidaka, Raaga, Mandala, Visarpini lakshana

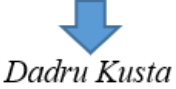

\section{ACTION OF DRUG AND OTHER PROCEDURE}

Table 3

\begin{tabular}{|c|c|c|}
\hline $\begin{array}{l}\text { S. } \\
\text { No }\end{array}$ & Dravya & Action \\
\hline 1 & Gandhak Rasayan vati Bhawan (2012) & Raktashodhak, Kandughana, rasayana \\
\hline 2 & Arogyavardhini vati Gune (2005) & $\begin{array}{l}\text { Dhatwagnivardhana, malashodhaka, } \\
\text { Pakwashyadushti nashka. }\end{array}$ \\
\hline 3 & Pachak vati Anupama (2014) & Amadosa nasaka \& Agni vardhaka \\
\hline
\end{tabular}




$\begin{array}{lll}4 & \text { Gandahrva haritaki vati Anupama (2014) } & \text { Anulomna, vatashulnashak } \\ 5 & \text { Mahamanjishtadhi Kashaya Ministry Health } & \begin{array}{l}\text { Varnya, Kushtaghana, raktaprasadnam, } \\ \text { Sonitstapana, rasayana }\end{array} \\ 6 & \begin{array}{l}\text { Avagahana (Triphala + Khadir + Nimba) } \\ \text { Tripathi (2011) }\end{array} & \begin{array}{l}\text { Kandughna, Tridoshghna, Vranya, } \\ \text { Raktashodhaka }\end{array}\end{array}$

\section{Nidana parivarjana}

Acharya sushruta also give importance to nidanparivarjanam. We advise patient to take bath daily, dry the body with towel, avoid prolong seating, if need prolong seating than take break of 2 min and stand till buttock part get dry, wash all cloths in Dettol mixed warm water.

\section{CONCLUSION}

Dadru like Skin disease difficult to cure by modern medicines. All steroid like medicine supresses the disease which later create worst state of disease. From the above case, it may be stated that Dadru can be completely cured by Ayurvedic treatment.

\section{REFERENCES}

Andhare, R. (2019). Management of Dadru Kushtha According To Ayurved : A Case Study, Iamj, 7(8), 14-27.

Anupama (2014). Gandharva Haritaki Churna Benefits, Ingredients, Dosage. Bhawan, K.G.A. (2012). Rasatantrasara Va Sidhaprayog Sangraha, Pratham Khanda, Kharaliya Rasayan -Page No. 225, 226.

Gune, G. P. (2005). Aushadhi Gundharma Shatra, Reprint, 208.

Ministry of Health and Family Welfare (2010). The Ayurvedic Pharmacopoeia Of India-Part-1, 113.

Puripanda, S. K. raju, M.B. Mallika, K.J. Tripathy, T.B. (2016). Understanding The Concept Of Sankramika Dadru Kusta- A Case Study : IJAPR | 4(8).81.

Sharangdhar (n.d.). Sharangdhar Pachak Vati 120 Tab, Packaging Size : 120Tabs, 2 Tabs Twice A Day After Meal.

Tripathi, B. (2011). Charak Samhita Of Maharshi Charak, Chukhambha Prakashan, Varanasi, Chikitsastan, Chapter 7, 252.

Tripathi, B. (2011). Charak Samhita, Maharshi Charak. Chikitsa-Stan;Kushta Chikitsa

Adhya: Chapter 7. Verse No.99. Varanasi : Chukhambha Prakashan, 320. Https://En.M.Wikipedia.Org/Wiki/Tinea_Cruris. (26 May 2020 : 12.20 Pm) 